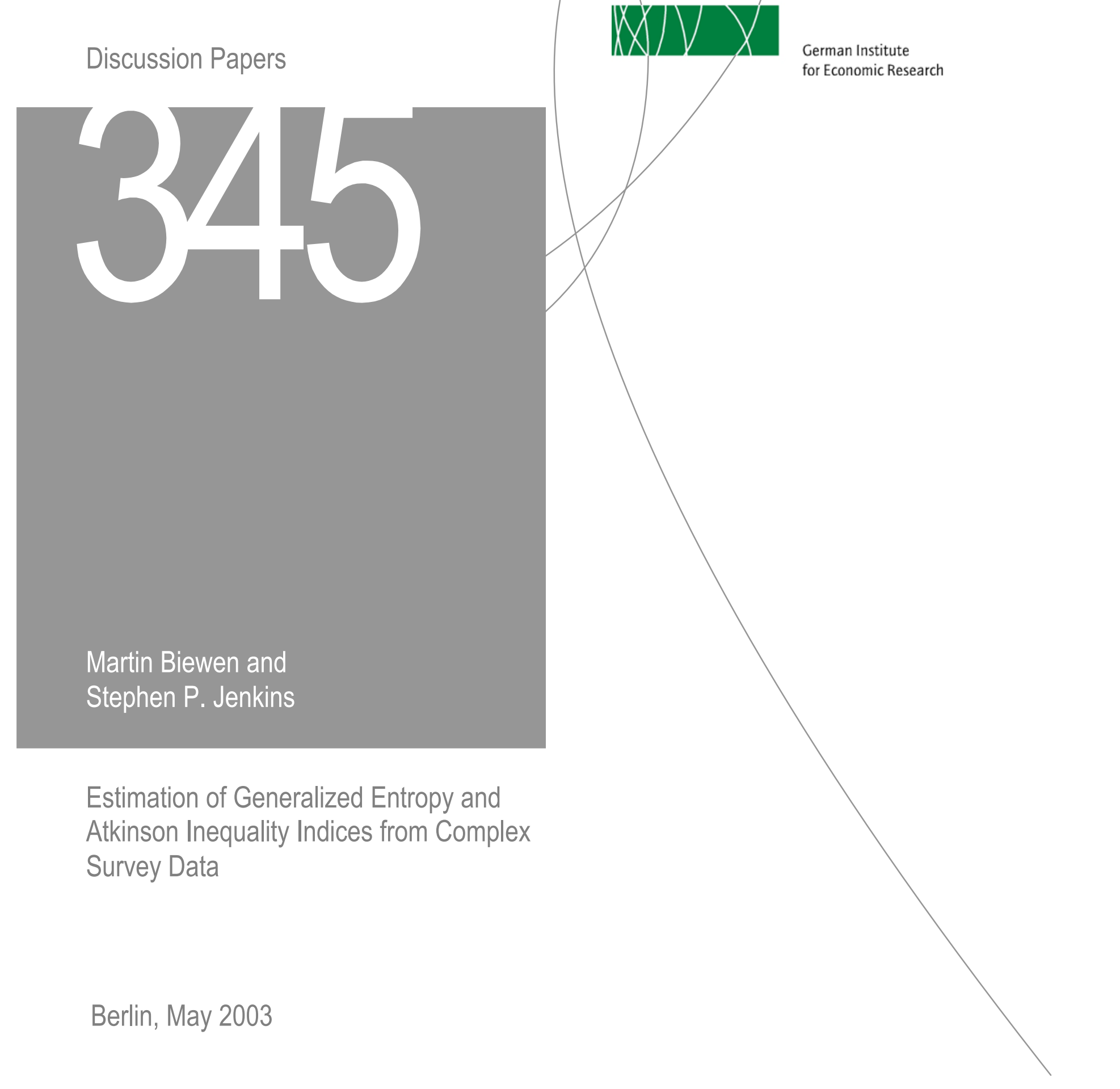


Opinions expressed in this paper are those of the author and do not necessarily reflect views of the Institute.

\section{DIW Berlin}

\section{German Institute}

for Economic Research

Königin-Luise-Str. 5

14195 Berlin,

Germany

Phone +49-30-897 89-0

Fax +49-30-897 89-200

www.diw.de

ISSN 1619-4535 


\title{
Estimation of Generalized Entropy and Atkinson Inequa- lity Indices from Complex Survey Data ${ }^{1}$
}

\section{Martin Biewen}

University of Mannheim

IZA, Bonn

DIW Berlin

\author{
Stephen P. Jenkins \\ ISER, University of Essex \\ IZA, Bonn \\ DIW Berlin
}

This version: 11 April 2003

\begin{abstract}
Applying a method suggested by Woodruff (1971), we derive the sampling variances of Generalized Entropy and Atkinson inequality indices when estimated from complex survey data. It turns out that this method also greatly simplifies the calculations for the i.i.d. case when compared to previous derivations in the literature. Both cases are illustrated with examples from the German Socio-Economic Panel Study and the British Household Panel Survey.
\end{abstract}

JEL-Classification: C14, D31

Keywords: Inequality, Statistical Inference, Complex Surveys

Correspondence:

Martin Biewen, Department of Economics, University of Mannheim, Verfügungsgebäude L7, 3-5, 68131 Mannheim, Germany, Fax: +49-621-1811841, biewen@rhein.vwl.uni-mannheim.de

\footnotetext{
${ }^{1}$ Financial support from the the Deutsche Forschungsgesellschaft (DFG) and core funding to ISER from the ESRC and the University of Essex is gratefully acknowledged.
} 


\section{Introduction}

Probability weighting, clustering, and stratification, are survey design features underlying much of the survey data that economists and others use. It is well known that these features have a potentially large impact on the sampling variability of statistics computed from such surveys. Nevertheless, they are rarely taken into account in practical work, the measurement of inequality being no exception. We derive estimates for the sampling variance of two commonly-used classes of inequality indices, the Generalized Entropy and the Atkinson family of indices, using the approach of Woodruff (1971). ${ }^{2}$ It turns out that Woodruff's method also greatly simplifies the computation of variance estimates in an i.i.d. framework when compared to previous derivations in the literature. In order to assess the error made by not taking into account clustering and stratification we apply our results to a sample extracted from the German Socio-Economic Panel (GSOEP) and the British Household Panel Survey (BHPS).

\section{Estimation from complex surveys}

Generalized Entropy and Atkinson inequality measures can be written, as we show below, as functions $I=f(T)$ of population totals $T=\left(T_{1}, \ldots, T_{K}\right)$. Population total $T_{k}, k=1, \ldots, K$ is given by the summation of an observational variable $t_{h i j k}$ over the different stages of the sampling design, i.e. $T_{k}=\sum_{n=1}^{L} \sum_{i=1}^{N_{h}} \sum_{j=1}^{M_{i}} t_{h i j k}$ where $L$ denotes the number of strata, $N_{h}$ the number of clusters in stratum $h$ and $M_{i}$ the number of individuals in cluster $i$. If the sampling design involves more than one stage of clustering it suffices to consider the first stage only (see e.g. Cochran, 1977). Replacing totals $T$ by their estimates $\hat{T}$, the index is then estimated as $\hat{I}=f(\hat{T})$ with $\hat{T}_{k}=\sum_{n=1}^{L} \sum_{i=1}^{n_{h}} \sum_{j=1}^{m_{i}} w_{h i j} t_{h i j k}$ where $n_{h}$ is the number of actually sampled first stage clusters and $m_{i}$ the number of actually sampled individuals in cluster $i$. The sampling weight of individual hij is given by $w_{h i j}$. Assuming that the sample is large enough that a Taylor approximation of $f(\cdot)$ holds, the variance of $\hat{I}$ can be approximated by the variance of the first order residual $\sum_{k=1}^{K}\left(\partial f(T) / \partial T_{k}\right) \hat{T}_{k}$. Woodruff (1971) observed that this variance can be easily determined by reversing the order of summation in the residual, i.e. $\operatorname{var}(\hat{I}) \approx \operatorname{var}\left(\sum_{n=1}^{L} \sum_{i=1}^{n_{h}} \sum_{j=1}^{m_{i}} w_{h i j}\left[\sum_{k=1}^{K}\left(\partial f(T) / \partial T_{k}\right) t_{h i j k}\right]\right)=\operatorname{var}(\hat{S})$. Note that $\hat{S}$ is of the

\footnotetext{
${ }^{2}$ An alternative but conceptually less straightforward approach to variance estimation in complex surveys is the estimating equations approach described in Binder (1983) and Binder and Patak (1994). It turns out that this approach leads to the same estimators derived here. Calculations are available from the authors on request.
} 
same form as the $\hat{T}_{k}$ s so that the problem is reduced to the estimation of the sampling variance of a total estimator for which well-known formulas exist (see Cochran, 1977, or Deaton, 1997). Using these formulas (and replacing $T$ by $\hat{T}$ in the derivative), the variance estimate for $\hat{I}$ is

$$
\widehat{\operatorname{var}}(\hat{I})=\sum_{n=1}^{L} \frac{n_{h}}{n_{h}-1} \sum_{i=1}^{n_{h}}\left(\sum_{j=1}^{m_{i}} w_{h i j} \tilde{s}_{h i j}-\frac{\sum_{i=1}^{n_{h}} \sum_{j=1}^{m_{i}} w_{h i j} \tilde{s}_{h i j}}{n_{h}}\right)^{2}
$$

with $\tilde{s}_{h i j}=\sum_{k=1}^{K}\left(\partial f(\hat{T}) / \partial \hat{T}_{k}\right) t_{h i j k}$.

If $y_{h i j}$ represents the income of individual hij, then population Generalized Entropy and Atkinson indices are given by

$$
\begin{aligned}
I_{G E}^{\alpha} & =\left(\alpha^{2}-\alpha\right)^{-1}\left[U_{0}^{\alpha-1} U_{1}^{-\alpha} U_{\alpha}-1\right], \quad \alpha \in \mathbb{R} \backslash\{0,1\} \\
I_{\text {Theil }} & =T_{1,1} U_{1}^{-1}-\log \left(U_{1} U_{0}^{-1}\right), \quad \alpha \rightarrow 1 \\
I_{M L D} & =-T_{0,1} U_{0}^{-1}+\log \left(U_{1} U_{0}^{-1}\right), \quad \alpha \rightarrow 0 \\
I_{A}^{\varepsilon} & =1-U_{0}^{-\varepsilon /(1-\varepsilon)} U_{1}^{-1} U_{1-\varepsilon}^{1 /(1-\varepsilon)}, \quad \varepsilon \geq 0, \quad \varepsilon \neq 1, \\
I_{A}^{1} & =1-U_{0} U_{1}^{-1} \exp \left(T_{0,1} U_{0}^{-1}\right), \quad \varepsilon \rightarrow 1
\end{aligned}
$$

for totals $U_{\alpha}=\sum_{n=1}^{L} \sum_{i=1}^{N_{h}} \sum_{j=1}^{M_{i}}\left(y_{h i j}\right)^{\alpha}$ and $T_{\alpha}=\sum_{n=1}^{L} \sum_{i=1}^{N_{h}} \sum_{j=1}^{M_{i}}\left(y_{h i j}\right)^{\alpha}\left(\log y_{h i j}\right)$. Note that $U_{0}$ is the population size. Estimates of these indices are obtained by replacing $U_{\alpha}, T_{\alpha}$ with $\hat{U}_{\alpha}=\sum_{n=1}^{L} \sum_{i=1}^{n_{h}} \sum_{j=1}^{m_{i}} w_{h i j}\left(y_{h i j}\right)^{\alpha}$ and $\hat{T}_{\alpha}=\sum_{n=1}^{L} \sum_{i=1}^{n_{h}} \sum_{j=1}^{m_{i}} w_{h i j}\left(y_{h i j}\right)^{\alpha}\left(\log y_{h i j}\right)$.

The corresponding variances are then given by substituting $\tilde{s}_{h i j}$ in (1) with

$$
\begin{aligned}
\tilde{s}_{h i j}^{G E} & =\frac{1}{\alpha} \hat{U}_{\alpha} \hat{U}_{1}^{-\alpha} \hat{U}_{0}^{\alpha-2}-\frac{1}{\alpha-1} \hat{U}_{\alpha} \hat{U}_{1}^{-\alpha-1} \hat{U}_{0}^{\alpha-1} \cdot y_{h i j}+\frac{1}{\alpha^{2}-\alpha} \hat{U}_{0}^{\alpha-1} \hat{U}_{1}^{-\alpha} \cdot\left(y_{h i j}\right)^{\alpha} \\
\tilde{s}_{h i j}^{T h e i l} & =\hat{U}_{1}^{-1} \cdot y_{h i j} \log y_{h i j}-\hat{U}_{1}^{-1}\left(\hat{T}_{1,1} \hat{U}_{1}^{-1}+1\right) \cdot y_{h i j}+\hat{U}_{0}^{-1} \\
\tilde{s}_{h i j}^{M L D} & =-\hat{U}_{0}^{-1} \cdot \log y_{h i j}+\hat{U}_{1}^{-1} \cdot y_{h i j}+U_{0}^{-1}\left(\hat{T}_{0,1} \hat{U}_{0}^{-1}-1\right) \\
\tilde{s}_{h i j}^{A} & =\frac{\varepsilon}{1-\varepsilon} \hat{U}_{1}^{-1} \hat{U}_{1-\varepsilon}^{\frac{1}{1-\varepsilon}} \hat{U}_{0}^{-\frac{1}{1-\varepsilon}}+\hat{U}_{0}^{\frac{-\varepsilon}{1-\varepsilon}} \hat{U}_{1-\varepsilon}^{\frac{1}{1-\varepsilon}} \hat{U}_{1}^{-2} \cdot y_{h i j}-\frac{1}{1-\varepsilon} \hat{U}_{0}^{\frac{-\varepsilon}{1-\varepsilon}} \hat{U}_{1}^{-1} \hat{U}_{1-\varepsilon}^{\frac{\varepsilon}{1-\varepsilon}} \cdot\left(y_{h i j}\right)^{1-\varepsilon}(10) \\
\tilde{s}_{h i j}^{1} & =\left(\hat{I}_{A}^{1}-1\right) \hat{U}_{0}^{-1}\left(1-\hat{U}_{0}^{-1} \hat{T}_{0,1}\right)+\left(1-\hat{I}_{A}^{1}\right) \hat{U}_{1}^{-1} \cdot y_{h i j}+\left(\hat{I}_{A}^{1}-1\right) \hat{U}_{0}^{-1} \cdot \log y_{h i j} .
\end{aligned}
$$

These variance estimators allow arbitrary correlations between observations below the firststage clusters. They are therefore an alternative to the estimators developed by Schluter and Trede (2002). 


\section{Application to the i.i.d. case}

In an i.i.d. framework the above indices are usually treated as follows. Income $x_{i}$ and weight $w_{i}$ of observation $i=1, \ldots, n$ are regarded as i.i.d. draws from a population $(x, w){ }^{3}$ The index in question can then be represented as a function $I=g(\mu)$ of population moments $\mu=E\left(X_{i}\right)$, where $X_{i}$ is a vector-valued function of $\left(x_{i}, w_{i}\right)$. It is estimated as $\hat{I}=g(\bar{X})$ and its sampling variance as $n^{-1} \nabla g(\bar{X})^{\prime} \widehat{\operatorname{var}}\left(X_{i}\right) \nabla g(\bar{X})$ (Cowell, 1989, or for the case without weights, Thistle, 1990). By contrast, Woodruff's method would yield a variance estimate $n^{-1 \widehat{\operatorname{var}}}\left(\nabla g(\bar{X})^{\prime} X_{i}\right)$. It is easy to see that both estimates are identical. However, Woodruff's method leads to much simpler expressions as the problem is reduced to estimating the sampling variance of a scalar. In particular, no covariances need to be computed. Defining $\mu_{\alpha}=E\left(w_{i} x_{i}^{\alpha}\right), \tau_{\alpha}=E\left(w_{i} x_{i}^{\alpha}\left(\log x_{i}\right)\right)$, $\hat{\mu}_{\alpha}=n^{-1} \sum_{i=1}^{n} w_{i} x_{i}^{\alpha}$ and $\hat{\tau}_{\alpha, \gamma}=n^{-1} \sum_{i=1}^{n} w_{i} x_{i}^{\alpha}\left(\log x_{i}\right)$, the sampling variances of the indices

$$
\begin{aligned}
I_{G E}^{\alpha} & =\left(\alpha^{2}-\alpha\right)^{-1}\left[\mu_{0}^{\alpha-1} \mu_{1}^{-\alpha} \mu_{\alpha}-1\right], \quad \alpha \in \mathbb{R} \backslash\{0,1\} \\
I_{T h e i l} & =\tau_{1,1} \mu_{1}^{-1}-\log \left(\mu_{1} \mu_{0}^{-1}\right), \quad \alpha \rightarrow 1 \\
I_{M L D} & =-\tau_{0,1} \mu_{0}^{-1}+\log \left(\mu_{1} \mu_{0}^{-1}\right), \quad \alpha \rightarrow 0 \\
I_{A}^{\varepsilon} & =1-\mu_{0}^{-\varepsilon /(1-\varepsilon)} \mu_{1}^{-1} \mu_{1-\varepsilon}^{1 /(1-\varepsilon)}, \quad \varepsilon \geq 0, \quad \varepsilon \neq 1, \\
I_{A}^{1} & =1-\mu_{0} \mu_{1}^{-1} \exp \left(\tau_{0,1} \mu_{0}^{-1}\right), \quad \varepsilon \rightarrow 1
\end{aligned}
$$

can therefore simply be estimated by substituting

$$
\begin{aligned}
\tilde{z}_{i}^{G E} & =\frac{1}{\alpha} \hat{\mu}_{\alpha} \hat{\mu}_{1}^{-\alpha} \hat{\mu}_{0}^{\alpha-2}-\frac{1}{\alpha-1} \hat{\mu}_{\alpha} \hat{\mu}_{1}^{-\alpha-1} \hat{\mu}_{0}^{\alpha-1} \cdot x_{i}+\frac{1}{\alpha^{2}-\alpha} \hat{\mu}_{0}^{\alpha-1} \hat{\mu}_{1}^{-\alpha} \cdot x_{i}^{\alpha} \\
\tilde{z}_{i}^{T h e i l} & =\hat{\mu}_{1}^{-1} \cdot x_{i} \log x_{i}-\hat{\mu}_{1}^{-1}\left(\hat{\tau}_{1,1} \hat{\mu}_{1}^{-1}+1\right) \cdot x_{i}+\hat{\mu}_{0}^{-1} \\
\tilde{z}_{i}^{M L D} & =-\hat{\mu}_{0}^{-1} \cdot \log x_{i}+\hat{\mu}_{1}^{-1} \cdot x_{i}+\mu_{0}^{-1}\left(\hat{\tau}_{0,1} \hat{\mu}_{0}^{-1}-1\right) \\
\tilde{z}_{i}^{A} & =\frac{\varepsilon}{1-\varepsilon} \hat{\mu}_{1}^{-1} \hat{\mu}_{1-\varepsilon}^{\frac{1}{1-\varepsilon}} \hat{\mu}_{0}^{-\frac{1}{1-\varepsilon}}+\hat{\mu}_{0}^{\frac{-\varepsilon}{1-\varepsilon}} \hat{\mu}_{1-\varepsilon}^{\frac{1}{1-\varepsilon}} \hat{\mu}_{1}^{-2} \cdot x_{i}-\frac{1}{1-\varepsilon} \hat{\mu}_{0}^{\frac{-\varepsilon}{1-\varepsilon}} \hat{\mu}_{1}^{-1} \hat{\mu}_{1-\varepsilon}^{\frac{\varepsilon}{1-\varepsilon}} \cdot x_{i}^{1-\varepsilon} \\
\tilde{z}_{i}^{1} & =\left(\hat{I}_{A}^{1}-1\right) \hat{\mu}_{0}^{-1}\left(1-\hat{\mu}_{0}^{-1} \hat{\tau}_{0,1}\right)+\left(1-\hat{I}_{A}^{1}\right) \hat{\mu}_{1}^{-1} \cdot x_{i}+\left(\hat{I}_{A}^{1}-1\right) \hat{\mu}_{0}^{-1} \cdot \log x_{i}
\end{aligned}
$$

for $\tilde{z}_{i}$ in

$$
\widehat{\operatorname{var}}(\hat{I})=\frac{1}{n(n-1)} \sum_{i=1}^{n}\left(w_{i} \tilde{z}_{i}-\frac{\sum_{i=1}^{n} w_{i} \tilde{z}_{i}}{n}\right)^{2} .
$$

Equation (22) has a similar structure to equation (1), but note that the weights are treated differently in the complex survey and i.i.d. cases. ${ }^{4}$

\footnotetext{
${ }^{3}$ If the distribution of household income among individuals is analyzed, then observational units $i$ are households and observations are replicated at the household level. In this case weights are $w_{i}=w_{i}^{\prime} w_{i}^{\prime \prime}$ with household size $w_{i}^{\prime}$ and sample weight $w_{i}^{\prime \prime}$. See Biewen (2002).

${ }^{4}$ For more discussion of different weighting concepts, see Cowell and Jenkins (2003).
} 


\section{Empirical illustration}

We contrast the variance estimators using data from the first waves of the German SocioEconomic Panel (SOEP Group, 2001), and the British Household Panel Survey (Taylor et al., 2002). ${ }^{5}$ The GSOEP and the BHPS are widely used to analyze the income distribution in these two countries. Moreover, they provide information on primary sampling units (clusters) and strata identification variables. The first wave of each survey was chosen to avoid complications of the panel design. We considered the distribution of income among individuals. Following convention, each person was assumed to receive the equivalent household income of the household to which she belonged. (The equivalence scale was the square root of household size.)

The survey estimates shown in column (1) of Table 1 take into account sampling weights, stratification and clustering. Replication of observations at the household level is automatically accounted for, as this represents a form of clustering below the first stage clusters. The i.i.d. estimates shown in column (2) only take into account sampling weights and replication of observations at the household level, but not stratification or clustering. A comparison of columns (1) and (2) indicates that ignoring clustering and stratification makes surprisingly little difference for these data sets. By contrast, the estimates shown in columns (3) and (4) suggest that taking into account the replication of observations at the household level is much more important. The results in column (3) ignore the replication of observations at the household level, whereas those in column (4) take it into account. (Both (3)and (4) ignore first-stage clustering and stratification.) For the German data, this leads to standard errors that are about twice as large. This shows that survey design can matter. However, the precise effect appears to depend on the survey analysed: corresponding estimates in columns (3) and (4) differ little when BHPS data are used, by contrast with the GSOEP case.

— Table 1 near here -

The fact that ignoring stratification and first-stage clustering has only a small impact might be interpreted as good news for practitioners using these data, or for those using surveys in which primary sampling unit and strata identification variables are not made available, but it is not clear that this empirical finding can be generalized. Whatever the case, our variance estimators provide a straightforward means by which researchers can accommodate a range of design effects in their analysis.

\footnotetext{
${ }^{5}$ Stata programs to compute the estimators are available from the authors on request.
} 


\section{References}

Biewen, M., 2002, Bootstrap Inference for Inequality, Mobility and Poverty Measurement. Journal of Econometrics 108, 317 - 342.

Binder, D.A., 1983, On the Variances of Asymptotically Normal Estimators from Complex Surveys, International Statistical Review 51, 293 - 300.

Binder, D.A. and Z. Patak, 1994, Use of Estimating Functions for Estimation From Complex Surveys, Journal of the American Statistical Association 89, 1035 - 1043.

Cochran, W.G., 1977, Sampling Techniques, 3d ed. (Wiley New York).

Cowell, F.A. , 1989, Sampling Variance and Decomposable Inequality Measures, Journal of Econometrics 42, 27 - 41.

Cowell, F.A. and Jenkins, S.P., 2003, Estimating Welfare Indices: Household Weights and Sample Design, in: Y. Amiel and J.A. Bishop, eds., Inequality, Welfare and Poverty: Theory and Measurement, Volume 9 (Elsevier Science, Amsterdam) 147 - 172, .

Deaton, A., 1997, The Analysis of Household Surveys: a Microeconometric Approach to Development Policy (Johns Hopkins University Press, Baltimore).

Schluter, C. and M. Trede, 2002, Statistical Inference for Inequality and Poverty Measures with Dependent Data, International Economic Review 43, 185 - 200.

SOEP-Group, 2001, The German Socio-Economic Panel (GSOEP) after more than 15 years Overview, in: Holst, E., D.R. Lillard and T.A. DiPrete, eds., Proceedings of the 2000 Fourth International Conference of German Socio-Economic Panel Study Users (GSOEP2000), Vierteljahreshefte zur Wirtschaftsforschung (Quarterly Journal of Economic Research) Vol. 70, 7 14.

Taylor, M., ed., 2002, British Household Panel Survey User Manual, Institute for Social and Economic Research, Colchester.

Thistle, P.D. , 1990, Large Sample Properties of Two Inequality Indices, Econometrica 58, 725 $-728$.

Woodruff, R.S., 1971, A Simple Method for Approximating the Variance of a Complicated Estimate, Journal of the American Statistical Association 66, 411 - 414. 


\section{Tables}

Table 1. Income inequality ${ }^{1}$ in West Germany (1984) and Britain (1991)

\begin{tabular}{|c|c|c|c|c|c|c|c|c|}
\hline \multirow[t]{2}{*}{ Index } & \multicolumn{2}{|c|}{ (1) Survey ${ }^{2}$} & \multicolumn{2}{|l|}{ (2) I.i.d. $^{3}$} & \multicolumn{2}{|c|}{ (3) Survey, i.i.d. ${ }^{4}$} & \multicolumn{2}{|c|}{ (4) Survey ${ }^{5}$} \\
\hline & estimate & std. err. & estimate & std. err. & estimate & std. err. & estimate & std. err. \\
\hline \multicolumn{9}{|c|}{ German Socio-Economic Panel ${ }^{6}$ (1984) } \\
\hline GE $(-1)$ & 0.4397 & 0.2971 & 0.3992 & 0.2573 & 0.4397 & 0.1333 & 0.4397 & 0.2978 \\
\hline MLD & 0.1339 & 0.0153 & 0.1338 & 0.0136 & 0.1339 & 0.0087 & 0.1339 & 0.0152 \\
\hline Theil & 0.1540 & 0.0276 & 0.1540 & 0.0246 & 0.1540 & 0.0187 & 0.1540 & 0.0276 \\
\hline $\mathrm{GE}(2)$ & 0.3673 & 0.1586 & 0.3502 & 0.1400 & 0.3673 & 0.1102 & 0.3673 & 0.1584 \\
\hline Atkinson $(0.5)$ & 0.0658 & 0.0079 & 0.0661 & 0.0071 & 0.0658 & 0.0052 & 0.0658 & 0.0079 \\
\hline Atkinson(1) & 0.1253 & 0.0134 & 0.1253 & 0.0119 & 0.1253 & 0.0076 & 0.1253 & 0.0133 \\
\hline Atkinson(1.5) & 0.2153 & 0.0477 & 0.2101 & 0.0419 & 0.2153 & 0.0219 & 0.2153 & 0.0478 \\
\hline Atkinson $(2)$ & 0.4679 & 0.1682 & 0.4439 & 0.1591 & 0.4679 & 0.0754 & 0.4679 & 0.1686 \\
\hline \multicolumn{9}{|c|}{ British Household Panel Survey ${ }^{7}$ (1991) } \\
\hline GE(-1) & 0.3656 & 0.0605 & 0.3627 & 0.0541 & 0.3656 & 0.0571 & 0.3655 & 0.0601 \\
\hline MLD & 0.1907 & 0.0051 & 0.1914 & 0.0050 & 0.1907 & 0.0030 & 0.1906 & 0.0050 \\
\hline Theil & 0.1779 & 0.0050 & 0.1784 & 0.0050 & 0.1779 & 0.0030 & 0.1779 & 0.0049 \\
\hline $\mathrm{GE}(2)$ & 0.2064 & 0.0085 & 0.2071 & 0.0086 & 0.2064 & 0.0054 & 0.2064 & 0.0085 \\
\hline $\operatorname{Atkinson}(0.5)$ & 0.0873 & 0.0022 & 0.0875 & 0.0022 & 0.0873 & 0.0013 & 0.0873 & 0.0021 \\
\hline Atkinson(1) & 0.1736 & 0.0042 & 0.1741 & 0.0041 & 0.1736 & 0.0025 & 0.1736 & 0.0041 \\
\hline Atkinson(1.5) & 0.2685 & 0.0084 & 0.2693 & 0.0082 & 0.2685 & 0.0058 & 0.2684 & 0.0082 \\
\hline Atkinson(2) & 0.4223 & 0.0404 & 0.4204 & 0.0363 & 0.4223 & 0.0381 & 0.4223 & 0.0401 \\
\hline
\end{tabular}

1 Income refers to monthly equivalent household net income distributed among individuals (equivalence scale $=$ square root of household size).

2 Survey estimator, individual data, weight = individual sample weight, accounting for clustering and stratification; replication of observations at household level automatically accounted for.

3 I.i.d. estimator, household data, weight $=$ household size ${ }^{*}$ household sample weight (thus accounting for replication of observations at household level), ignoring clustering and stratification.

4 Survey estimator, individual data, weight = individual sample weight, ignoring clustering, stratification and replication of observations at household level (identical to i.i.d. estimator, individual data, weight $=$ individual sample weight).

5 Survey estimator, individual data, weight = individual sample weight, ignoring clustering and stratification but accounting for replication of observations at household level (households are interpreted as clusters).

6110 strata, 516 clusters, 4232 households, 9441 individuals.

775 strata, 250 clusters, 4814 households, 11616 individuals. 